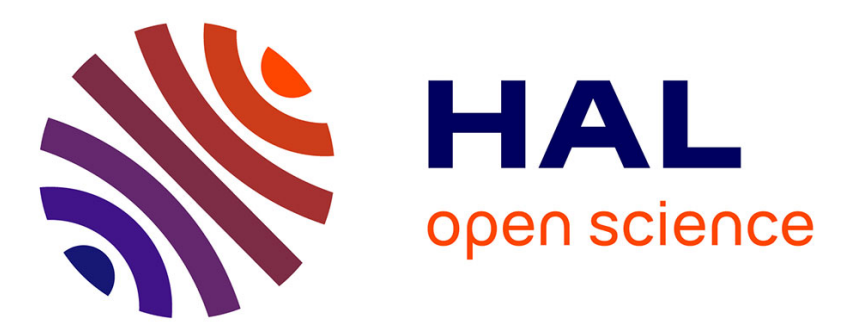

\title{
Framework for clathrate hydrate flash calculations and implications on the crystal structure and final equilibrium of mixed hydrates
}

\author{
Baptiste Bouillot, Jean-Michel Herri
}

\section{- To cite this version:}

Baptiste Bouillot, Jean-Michel Herri. Framework for clathrate hydrate flash calculations and implications on the crystal structure and final equilibrium of mixed hydrates. Fluid Phase Equilibria, 2016, 413, pp.184-195. 10.1016/j.fluid.2015.10.023 . hal-01220223

\section{HAL Id: hal-01220223 \\ https://hal.science/hal-01220223}

Submitted on 29 Jul 2016

HAL is a multi-disciplinary open access archive for the deposit and dissemination of scientific research documents, whether they are published or not. The documents may come from teaching and research institutions in France or abroad, or from public or private research centers.
L'archive ouverte pluridisciplinaire HAL, est destinée au dépôt et à la diffusion de documents scientifiques de niveau recherche, publiés ou non, émanant des établissements d'enseignement et de recherche français ou étrangers, des laboratoires publics ou privés. 


\title{
Framework for Clathrate Hydrate Flash Calculations and Implications on the Crystal Structure and Final Equilibrium of Mixed Hydrates
}

\author{
Baptiste Bouillot ${ }^{\mathrm{a}, *}$, Jean-Michel Herri ${ }^{\mathrm{a}}$ \\ ${ }^{a}$ École Nationale Supérieure des Mines de Saint-Etienne \\ Centre SPIN - LGF UMR CNRS 5307 \\ 158, cours Fauriel, 42023 Saint-Etienne, FRANCE
}

\begin{abstract}
Clathrate hydrates, usually called gas hydrates, are compounds of great interest in oil industry, as well as in gas separation and storage, water purification etc... Like many other compounds that phase change, in this case from liquid water to non stoichiometric crystalline compound, modeling is required to understand and optimize the processes that involve them.

Therefore, the classic thermodynamic equilibrium model is combined with mass balance calculations during gas hydrate crystallization. Two frameworks for performing clathrate hydrate thermodynamic flash calculations at constant volume are presented and compared to experimental results at low crystallization rate. The inputs are the quantity of mass (water and gas molecules), and the volume. The variable is temperature (three phases thermodynamic flash at a given temperature), while the volume is kept constant.

The first framework suggests that the hydrate phase is growing at local thermodynamic equilibrium, without any reorganization of its content of the occupancy of the cavities. In the second framework, the hydrate phase can reorganize itself during growth (locally or completely). These frameworks are investigated, as well as the impact of the Kihara parameters uncertainties.

These frameworks calculate well the final pressure, hydrate composition. In addition, the hydrate volume and mole amount in each phase is provided with reasonable accuracy. Note: uncertainties on the final pressure and hydrate volume are below 5\%. Moreover, the results are quite sensitives to
\end{abstract}

\footnotetext{
*bouillot@emse.fr
} 
the value of the Kihara parameters, demonstrating the importance of their values for a given computer code.

This work provides a more reallistic and comprehensive view of gas hydrate crystallization.

Keywords: Thermodynamics, Crystallization, Clathrate hydrates, Flash calculations

2

\section{Introduction}

Clathrate hydrates (also called gas hydrates) are crystalline water-based solids that resemble water ice, in which small molecules (typically gases) are trapped inside cavities of hydrogen bonds. They form under high pressure (few to tens Mpa) at low temperature (usually under $20{ }^{\circ} \mathrm{C}$ ), and cover vast areas of the ocean floor for example. These gas molecules, or guest molecules, are captured in these cavities. These cages are stabilized by van der Waals forces. Thanks to these forces, the clathrate hydrate structure is made possible. Note that recently Falenty et al.[1] managed to form empty SII clathrate hydrates at $145 \mathrm{~K}$.

As crystals, different polymorphic forms are known and were studied in the past [2]. There are three main structures: SI, SII and SH, composed of different kind of cavities, surrounded by differing polyhedra sharing faces. Jeffrey [3] suggested the nomenclature $e^{f}$ to describe the polyhedra (where $e$ is the number of edges, and $f$ the number of faces). The polymorphic forms exhibit different densities with differences in terms of thermodynamic equilibrium. Most important, the kind of guest molecules impacts the polymorphic form to be crystallized. In addition, for a given gas mixture, the occupancy of the cavities is not stoichiometric.

Clathrate hydrates are an crucial issue in many fields, from oil \& gas industry $[4,5]$, to carbon capture and storage [6], air conditioning [7], or even planetary science [8]. They have an enormous potential as an energy resource because of the tremendous quantity of methane trapped within them[9]. Understand that hydrate plugs in deep-sea pipelines probably pushed the worldwide study of clathrate hydrates into being. Hydrate management, or "flow assurance", in the petroleum industry is a complex technical challenge. Hydrate plugs can have catastrophic consequences, stopping the oil production for days or weeks, with the difficulty of dissociating the hydrate crystals. There are many solutions to this problem, such as kinetic or thermodynamic 
additives, or just employing avoidance techniques[10]. In the case of kinetic additives, the aim is to slow down the hydrate nucleation and growth (KHI, for "Kinetic Hydrate Inhibitor"), or to avoid agglomeration (AA, for "Anti-Agglomerant"). In thermodynamic additives (usually alcohols, such as methanol), the objective is to change the thermodynamic equilibrium itself, and avoid the hydrate zone.

To investigate hydrate avoidance, and/or thermodynamic inhibitors, both the understanding of the thermodynamics and the crystallization mechanisms of clathrate hydrates are required. While a thermodynamic model exists to simulate clathrate hydrate liquid-solid equilibrium, there is a major issue in modeling complex situations (such as flow assurance). Moreover, the quantity of kinetic inhibitors to avoid the hydrate formation in a pipe is dependent on the volume of crystal to be formed. So, there is a request to know "when", and "how many".

The thermodynamic modeling gives the final state of a system. Usually, this final equilibrium is enough for the modeling of chemical processes, since the thermodynamic path does not matter. In the case of clathrate hydrates, non-stoichiometric compounds are studied, and there is a question about the role of the thermodynamic path. It could matter in this specific case since different mechanisms need to be considered. In our study, many parameters are taken into account: gas consumption that changes the gas composition, and hence the thermodynamic equilibrium; molecules enclathration in the crystal caracterized by the occupation of cavities (driven by thermodynamics or kinetics[11]).

Hence, it is crucial to understand clathrate hydrate crystallization to model the local change over time of the hydrate composition during crystal growth. At the end of the crystallization, whatever the topic (flow assurance, carbon capture and storage, planetary science...), the modeling of clathrate hydrate equilibrium and crystallization is key to develop process simulation and sizing. Especially beacause the volume of the final crystal phase and the amount of gas captured is a essential result to predict. For this purpose, the methodology can be a thermodynamic or kinetic. A kinetic model, incorporating crystal growth, was recently suggested by Herri and Kwaterski[12]. More recently, a kinetic flash algorithm has been suggest by Boesen et al.[13], based on the Skovborg model[14].

In the present approach, a thermodynamic method was chosen, combined with mass balance calculation, that is to say a thermodynamic flash approach for clathrate hydrates. For this endeavor, some assumptions are needed to 
take into account the molecules' enclathration. Either the hydrate is growing at local thermodynamic equilibrium, or the whole crystal phase is at thermodynamic equilibrium. So, the two assumptions are considered within a flash calculation.

Our objective is to present two frameworks for the clathrate hydrate thermodynamic flash calculations at constant volume. By presenting to differing frameworks, three kinds of calculations are available: two at local equilibrium, and one at overall equilibrium. Then, the resulting simulations are compared to an experimental reference case from another study[11] that was performed at slow crystallization rate (to avoir kinetics effects). The aim is to model the hydrate volume in the end of the crystallization, and final pressure far from equilibrium. Another objective is to adjust the classic thermodynamic parameters (usually Kihara parameters) in the case where the equilibrium pressure is not sufficient.

This paper is organized as follows: section 2 explains further the theory involved. Section 3 details the modeling approach with the two thermodynamic frameworks utilized. Section 4 then presents the simulation results in some details. In additino, a parametric study is suggested. Finally, the conclusion and some future works are discussed.

\section{Theory}

\subsection{Calculation of VLE equilibrium}

\subsubsection{Calculating the vapor phase density}

The vapor phase density and fugacity coefficient are usually calculated using an equation of state $\left(f\left(P, V_{m}, T\right)=0\right)$. Among the numerous equations of state available, the Soave-Redlich-Kwong (SRK)[15] is known to be a good choice for density calculation for vaporized small hydrocarbon molecules[16]. This equation was used with the parameters from Danesh[16].

Since we consider a constant volume, the knowledge of the vapor phase density defines the mass (or number of mole) of the initial gas phase $(=$ $\left.P V / Z_{S R K} R T\right)$.

\subsubsection{Calculating gas solubility in water}

The gas molecule solubility into the water phase can be expressed from the equality of the chemical potentials (or the fugacities) of each molecules in the two phases (liquid and vapor). Considering a hydrocarbon gas mixture, a standard $\gamma^{\infty} / \phi$ approach is usual[17]. The $\gamma^{\infty}$ account for the activity 
coefficient at infinite dilution (Henry's law approach, introducing Henry's constants), while $\phi$ is the fugacity coefficient (calculated using an equation of state). Since the vapor-liquid equilibrium takes into account the hydrocarbon molecules only, the water molecule in the vapor phase is neglected. This leads to the following equation:

$$
f^{V}=f^{L_{w}} \leftrightarrow P \phi_{i} x_{i}^{L_{w}}=x_{i}^{L_{w}} K_{H, i, w}\left(T, P_{w}^{\sigma}\right) \exp \left(\frac{P V_{m, i}^{\infty, L_{w}}}{R T}\right)
$$

where $K_{H, i, w}$ is the Henry's constant of molecule $i$ in liquid water $L_{w}$, and $V_{m, i}^{\infty, L_{w}}$ the partial molar volume of the molecule $i$ at infinite dillution.In the same manner as our previous work[18], an avergae value of $V_{m, i}^{\infty, L_{w}}=$ $32 \mathrm{~cm}^{3} \mathrm{~mol}^{-1}$ was used[19], and the Henry's constant were calculated from an empirical equation[20].

\subsection{Thermodynamic equilibrium of clathrate hydrates}

Phase equilibria are described by the equality of chemical potentials, $\mu$, in each phase. In the case of clathrate hydrates thermodynamic equilibrium, this is the equality of water in both liquid and hydrate phase :

$$
\mu_{w}^{H}=\mu_{w}^{L}
$$

Actually, clathrate hydrate equilibrium is usually described by the van der Waals and platteuw model [21]. This model is based on classic thermodynamics for the water liquid phase (Gibbs-Duhem equation), and on statistical thermodynamics for the hydrate phase, based on the following assumptions:

- Each cavity contain one guest molecule at best,

- the interaction between the guest molecule and the cavity (water molecules) can be described by a pair potential function of the pair gas-molecule,

- the cavities are perfectly spherical,

- The guest molecules do not deform cavities,

- There is no interactions between the guest molecules.

The van der Walls and Platteuw model also writes the phase equilibrium with an hypothetic reference state ( $\beta$ state) corresponding to the hydrate phase with empty cavities: 


$$
\Delta \mu_{w}^{H-\beta}=\Delta \mu_{w}^{L-\beta}
$$

where $\Delta \mu_{w}^{H-\beta}$ and $\Delta \mu_{w}^{L-\beta}$ are the difference between the chemical potential of the hydrate phase (respectively the liquid phase), and the chemical potential of the hypothetic $\beta$ phase.

\subsubsection{Liquid phase}

As previously mentioned, the second part of equation 3 is expressed from classic thermodynamics, while the first part is expressed from statistical thermodynamics. Hence $\Delta \mu_{w}^{L-\beta}$ is written using Gibbs-Duhem equation as follows:

$\Delta \mu_{w}^{L-\beta}=T \frac{\left.\Delta \mu_{w}^{L-\beta}\right|_{T^{0} P^{0}}}{T^{0}}-T \int_{T^{0}}^{T} \frac{\left.\Delta h_{w, m}^{L-\beta}\right|_{P^{0}}}{T^{2}} \mathrm{~d} T+\left.\int_{P^{0}}^{P} \Delta \nu_{w, m}^{L-\beta}\right|_{T^{0}} \mathrm{~d} P-\left.R T \ln a_{w}^{L}\right|_{T, P}$

where $\left(T^{0}, P^{0}\right)$ are reference temperature and pressure, $\left.\Delta \mu_{w}^{L-\beta}\right|_{T^{0} P^{0}}$ is the chemical potential difference of water in the liquid phase and water in the hydrate free cavity phase at the reference state, $\left.\Delta h_{w, m}^{L-\beta}\right|_{P^{0}}$ the molar enthalpy difference between the liquid and $\beta$ phase, $\left.\Delta \nu_{w, m}^{L-\beta}\right|_{T}$ the molar volume difference between the liquid and the $\beta$ phase, and $\left.a_{w}^{L}\right|_{T, P}$ the activity of water in the liquid phase at $(T, P)$.

Since the water activity is expressed with the activity coefficient $a_{w}=$ $\gamma_{w} x_{w}^{L}$ and only "pure" water is involved, it is usual to consider the ideal case $\left(\gamma_{w}=1\right)$ (no additives, only gas molecules dissolved into the aquaeous phase, showing weak deviation from ideality).

$\left.\Delta \mu_{w}^{L-\beta}\right|_{T^{0} P^{0}}$ and $\left.\Delta h_{w, m}^{L-\beta}\right|_{P^{0}}$ are thermodynamic properties of the liquid phase compared to reference $\beta$ phase. As for any thermodynamic model based on activity coefficient formalism, these thermodynamic properties are essential. Many values can be found in the litterature according to various authors. Table 1 gives some of these values.

$\left.\Delta \nu_{w, m}^{L-\beta}\right|_{T^{0}}$ is a first order parameter that has been measured with high accuracy by von Stackelberg [22] via X-Ray diffraction.

$\left.\Delta h_{w, m}^{L-\beta}\right|_{P^{0}}$ is a first order parameter whose expression has been refined by Sloan $[20,2]$, leading to:

$$
\left.\Delta h_{w, m}^{L-\beta}\right|_{P^{0}}=\left.\Delta h_{w, m}^{L-\beta}\right|_{T^{0}, P^{0}}+\left.\int_{T^{0}}^{T} \Delta C_{p_{w, m}}^{L-\beta}\right|_{P^{0}} \mathrm{~d} T
$$


where $\Delta C_{p_{w, m}}^{L-\beta}$ is the molar calorific capacity difference between liquid water and hydrate $\beta$ phase, and can be expressed as:

$$
\left.\Delta C_{p_{w, m}}^{L-\beta}\right|_{P^{0}}=\left.\Delta C_{p_{w, m}}^{L-\beta}\right|_{T^{0}, P^{0}}+b_{p, w}^{L-\beta}\left(T-T^{0}\right)
$$

Parameters $\left.\Delta C_{p_{w, m}}^{L-\beta}\right|_{T^{0}, P^{0}}$ and $b_{p, w}^{L-\beta}\left(T-T^{0}\right)$ have been calculated by Sloan et al. [2] (see Table 2).

The final equation (from equation 4) is:

$$
\begin{gathered}
\Delta \mu_{w}^{L-\beta}=T \frac{\Delta \mu_{w}^{L-\beta} \mid T^{0}, P^{0}}{T^{0}}+\left(b_{p, w}^{L-\beta} T^{0}-\Delta C_{p_{w, m} \mid T^{0}, P^{0}}^{L-\beta}\right)-T \ln \frac{T}{T^{0}}+\frac{1}{2} b_{p, w}^{L-\beta} T\left(T^{0}-T\right)+ \\
\left(\left.\Delta h_{w, m}^{L-\beta}\right|_{\mid T^{0}, P^{0}} ^{L}+\left(b_{p, w}^{L-\beta} T^{0}-\Delta C_{p_{w, m}{ }^{L-\beta}, T^{0}, P^{0}}^{L-}\right) T^{0}-\frac{1}{2} b_{p, w}^{L-\beta} T^{0^{2}}\right)\left(1-\frac{T}{T^{0}}\right)+ \\
\Delta \nu_{w, m}^{L-\beta}\left(P-T^{0}\right.
\end{gathered}
$$

In a previous study [18], the parameters of Handa and Tse [23] were found to be the best set for the modeling of pure clatrate hydrates. That is why they were chosen.

\subsubsection{Hydrate phase}

The second part of equation 3 deals with statistical thermodynamic development of hydrate chemical potential. In the van der Waals and Platteuw model [21], this potential is expressed with the occupancy factor $\theta_{j}^{i}$ of molecule $j$ in cavity $i$ of the crystal, therefore:

$$
\Delta \mu_{w}^{H-\beta}=R T \sum_{i} \nu_{i} \ln \left(1-\sum_{j} \theta_{j}^{i}\right)
$$

where $\nu_{i}$ is the number of type $i$ cavities per mole of water. In this model, the occupancy factor is expressed from Langmuir kind approach:

$$
\theta_{j}^{i}=\frac{C_{j}^{i} f_{j}(T, P)}{1+\sum C_{j}^{i} f_{j}(T, P)}
$$

where $C_{j}^{i}$ is the Langmuir constant of molecule $j$ in cavity $i$, and $f_{j}(T, P)$ is the fugacity of component $j$ at temperature $T$ and pressure $P$ in the liquid phase. Usually, this fugacity is to be equal to the fugacity in the gas phase since there are three phases at equilibrium in hydrates experiments (gas, 
aquaeous and hydrate). In these cases, a standard equation of state can be used for the calculations.

Then, the Langmuir constant is calculated from the interaction potential:

$$
C_{j}^{i}=\frac{4 \pi}{k_{B} T} \int_{0}^{R-a} \exp \left(-\frac{w(r)}{k_{B} T}\right) r^{2} d r
$$

The integration of potential interaction $w$ is calculated from 0 (surface of gas molecule) to $R-a$, the distance between the molecule and the cavity. In clathrate hydrate field, the Kihara potential is used [24], written:

$$
w(r)=2 z \epsilon\left[\frac{\sigma^{12}}{R^{11} r}\left(\delta^{10}+\frac{a}{R} \delta^{11}\right)-\frac{\sigma^{6}}{R^{5} r}\left(\delta^{4}+\frac{a}{R} \delta^{5}\right)\right]
$$

with

$$
\delta^{N}=\frac{1}{N}\left[\left(1-\frac{r}{R}+\frac{a}{R}\right)^{-N}-\left(1+\frac{r}{R}+\frac{a}{R}\right)^{-N}\right]
$$

Parameters $\epsilon, \sigma$ and $a$ are the so-called Kihara parameters. $\epsilon$ corresponds to the maximum attractive potential, $\sigma$ the distance from the cavity center, and $a$ the hard-core radius. They are fitted for one molecule to experimental data of pure gas hydrates (except for $a$ that is usually a known geometric parameter). To do so, a simple root mean square error (RMSE) minimization of the deviation function is performed from $N$ PT data $(N>2)$ :

$$
F(\epsilon, \sigma)=\sqrt{\frac{\sum\left(P_{\text {pred }}-P_{\text {exp }}\right)^{2}}{N}} \rightarrow 0
$$

where $P_{\text {pred }}$ and $P_{\text {exp }}$ are the predicted and experimental pressure (respectively) at temperature $T$.

Of course, many Kihara parameters can be found in the literature, depending on authors, thermodynamic properties and experimental data used. More details on Kihara parameters optimization, and differences between authors, can be found in a previous study [18].

\subsubsection{Kihara parameters optimization limits}

Kihara parameters optimization is a tricky part in the thermodynamic modeling of clathrate hydrates. As stated previously, there are as many sets of parameters as there are authors. There are different issues to explain this observation. 
First, the quality of the thermodynamic parameters (table 1) can play an important role[18].

Concerning again optimization, the predicted versus experimental equilibrium pressure is oftenly used. To avoid non-thermodynamic equilibria, only pure gas hydrates should be considered. But sometimes, this is not enough to find a global minimum to the deviation function. In these cases, gas mixtures have to be considered, and the deviation function is based not only on the pressure, but also on the hydrate composition as follows:

$$
F^{\prime}(\epsilon, \sigma)=\sqrt{\frac{\sum\left(x_{p r e d}^{H}-x_{e x p}^{H}\right)^{2}}{N}} \rightarrow 0
$$

The first problem with this calculation is that the literature data rarely gives the mixed hydrate composition, especially at total dissociation point (hard to obtain and to measure since the hydrate volume is then close to zero).Then, and this is the main issue, the experimental data obtained and published in the literature might not be at thermodynamic equilibrium (quick crystallizations).

Indeed, in a previous study, it was observed that the experimentally obtained thermodynamic three phases equilibrium (vapor-liquid-hydrate, or VLHE) is not systematic [11]. If the equilibrium pressure of pure gas hydrates corresponds exactly to the thermodynamic equilibrium, it is not always the same for mixed hydrates (clathrate hydrate from gas mixtures). When several gases are involved, kinetics considerations arise. For example, the kinetics of gas dissolution in the water phase suggest a different driving force for the crystallization than the one predicted by pure thermodynamics. Then, the kinetics of gas enclathration into the hydrate structure could diverge from thermodynamics. Certainly, thermodynamically, various configurations of the occupancy of the cavities can stabilize the crystalline structure. In theory, the occupancy factor depends on the langmuir constants and the gas fugacities in the aqueous phase. All the previous kinetics considerations question the validity of a thermodymic model when the experiments are performed at high driving force (far from equilibrium).

In our previous work, it appeared that the driving force of the crystallization impacts the "thermodynamic path" during the crystallization. As a result, in the end of the crystallization, the hydrate phase composition, the final pressure and the final hydrate volume, were not the same at low and high driving forces[11] (severals bars between a slow and a quick crystallization). 
To take account of kinetics, a novel model was recently suggested by Herri and Kwaterski [8]. In their model, the rate of enclathration of the gas molecules into the hydrate crystal is considered in the langmuir constant (instead of integration an interaction potential, see equation 10).

In the next section, a framework to perform flash calculations with the classic thermodynamic modeling of gas hydrates is presented. The objective is to take into account the possible non-stoichiometric composition of the hydrate crystals, and to study more precicely the mechanisms of the hydrate crystallization process, and the influence of the uncertainties on the Kihara parameters. Moreover, it can give another criterion for Kihara parameters optimization from pure gas hydrate: the hydrate volume.

\section{Modeling Clathrate hydrate crystallization}

\subsection{Flash calculation frameworks}

\subsubsection{Classic flash calculations and hydrate approaches}

The basic of a thermodynamic flash (or "flash") is to determine, from an initial given state, the composition and mass in each phase, by taking care of the Gibbs phase rule.

In a usual flash calculation, all phases are at equilibrium and their composition is homogeneous. The flash algorithm is composed of two loops:

- the thermodynamic equilibrium (equality of chemical potentials/fugacities by the mean of partition coefficients),

- mass balance calculation from partition coefficients.

So, there is one calculation loop on a thermodynamic intensive property (usually temperature or pressure), and one loop concerning the mass balance (an extensive property such as the mass of the components). According to the Gibbs phase rule, the three phases equilibria allow one less variable than the simple two phases flash equilibria. In this article, a thermodynamic flash at constant volume is considererd (the volume of the reactor in a typical batch experiment).

Hydrate flash calculation is a tricky problem since the hydrate phase composition is non-stoichiometric. This is due to the gas consumption leading to pressure variations and the gas/liquid composition change during crystallization (batch experiments). 
In the case of clathrate hydrates, the composition of the solid phase is, $a$ priori, not homogeneous. That is why a partition coefficient cannot be used to describe the whole solid phase. The thermodynamic calculation can only describe the composition locally in time. Towards the end, the calculation has to be performed step by step, by considering successives "classic flash calculations", corresponding to the crystal growth. Figure 1 illustrates this process. In the end, the hydrate crystal shows an occupation gradient of the cavity by the gas molecules. Hence, this flash calculation can be considered as a crystal growth at successives equilibria.

Of course, this model has as hypothesis that the local composition at the core of the hydrate crystal is not at equilibrium with the liquid phase. Only the last layer is. This procedure is then called Flash calculation framework without hydrate phase reorganization (see next section).

This hypothesis is fundamental, and corresponds to a mass balance for a given amount of crystallized hydrate. As there is a discretization of the crystal growth step by step, this hydrate flash is then a succession of usual thermodynamic flash algorithms instances.

There is another approach possible: change the previous hypothesis. It is conceivable that, at the end of each step during the crystal growth, the crystal reorganizes itself and reaches thermodynamic equilibrium. Thus, the last crystal layer is at thermodynamic equilibrium with the surrounding liquid phase. Furthermore, it is possible to obtain a homogeneous final crystal by not discretizing the crystal growth. Our second approach is also presented and discussed after under the name Flash calculation framework with hydrate phase reorganization (see also figure 5 further on in the text).

\subsubsection{Flash calculation framework without hydrate phase reorganization}

Our first approach concerns the model with no solid phase reorganization. Figure 1 illustrates this method. The first crystals appear in the bulk at thermodynamic equilibrium with the liquid phase at the begining of the nucleation. The occupancy of the cavities of this new hydrate phase (hydrate nuclei) is controlled by thermodynamics and can be calculated from van der Waals and Platteuw model.

Figure 1 also shows a discretization of the crystal growth. The more iterations there are, the more the growth is continuous. The main objective of this hydrate growth modeling is to follow the thermodynamic equilibrium curves, as shown on figure 2, and later in figure 7 . On the former figure, there is not only one thermodynamic equilibrium curve since the gas in consummed. 
As the gas is consummed, the pressure decreases, and the gas compositions in fluid phases change along the crystallization. Since the gas hydrate equilibrium curve (PT) depends on the gas composition, the crystal growth goes through many steps until the final composition is reached. The number of iterations (discretization) is important since it fixes the amount of gas in the hydrate phase at each step. It is a growth model locally at thermodynamic equilibrium (on the crystal surfaces).

The figures 2 and 3 illustrate the whole framework for the hydrate flash. The framework is define by the starting conditions:

- initial temperature $T_{i}$,

- initial pressure $P_{i}$,

- initial gas composition $z_{i}$,

- the total fixed volume (= initial gas volume, single phase before liquid injection, or "reactor volume") $V^{R}$,

- initial mass of water $m_{i}$,

These inputs allow for the calculation of the mass of each compound in each phase (liquid-gas). The total mass of the gas phase is calculated with the SRK equation of state. This corresponds to point $A$ on figure 2, which is also an initial liquid-vapor thermodynamic flash calculation (VLE thermodynamic flash).

This initial VLE thermodynamic flash is at constant temperature and volume (flash TV). The SRK equation of state is used to model the vapor phase, while a Henry's law approach for the gas solubility is considered (VLE, see section 2.1). More information about thermodynamic flash calculations can be found in the literature[25].

Then, another two inputs of the framework are essential:

- the final temperature $T_{f}$ (= final state),

- the number of iterations $n$ to reach the final state.

Gibbs phase law for three phases equilibria gives one degree of freedom less than for simple VLE flash. Here, the temperature $T_{f}$ is the variable. The final temperature imposes the final state of the calculation (the temperature is the 
only variable). The number of iterations corresponds to the discretization of the hydrate flash, via decreasing increments of temperature $(\Delta T)$.

The second step of the flash framework is to determine the temperature at which the liquid-hydrate thermodynamic equilibrium (LHE) is reached (point $B$ ). It is a VLE flash that also satisfy the LHE equation. The equilibrium temperature $T_{B}$ is determined from the predicted pressure of the VLE equilibrium and LHE equilibrium $\left(P^{V L E}=P^{L H E}\right)$

From this point, the temperature step is calculated from the number of iterations: $\Delta T=\left(T_{B}-T_{f}\right) / n$. The higher $n$ is, the more continuous the growth is. The lower $n$ is, the more the driving force is significant in the model.

Then, the crystal growth begins. The temperature is decreased step by step $(\Delta T)$ until the final temperature $T_{f}$. Both thermodynamic equilibria (VLE and LHE) have to be checked before going any further. The VLE is checked flash calculation prior to the LHE calculation. This VLE flash provides an equilibrium pressure $P^{V L E}$. Then, the LHE is calculated, providing another equilibrium pressure $P^{H}$. The error between $P^{V L E}$ and $P^{H}$ is the criterion of the algorithm. The variable that is used to minimize this error is the mass (volume) of water that has crystallized into the hydrate phase. This mass has consequences on the fluid volume (and also the VLE flash) and the LHE since the amount of gas molecules in each phase is affected (through the occupancy factor $\theta_{j}^{i}$ ).

At each step, a given mass of liquid water is transfered to the hydrate phase $\left(d m_{w}^{i}\right)$. As a consequence, the total volume of the hydrate phase $\left(V^{H}\right)$ is increased. This volume is calculated from the density of the empty clathrate. These densities $\left(790 \mathrm{~kg} / \mathrm{m}^{3}\right.$ for structure I, and $785 \mathrm{~kg} / \mathrm{m}^{3}$ for structure II) can be obtained from lattice parameters of each structure, neglecting temperature dependency. Sloan and Koh[2] also suggested a formula to calculate hydrate density. In the end, the hydrate volume crystallized is:

$$
V^{H}=\frac{\sum_{i} d m_{w}^{i}}{\rho^{H-\beta}}
$$

In order to determine the gas consumption toward the hydrate phase, the occupancy of the cavities $\left(\theta_{j}^{i}\right)$ is calculated from van der Waals and Platteuw model.

At this point, the amount of matter taken from the fluid phase, and added to the solid phase, is known. A new VLE flash is performed on the fluid phases. This provides $P^{V L E}$. The new gas composition in the liquid phase 
is also known, and the LHE can be checked by determining the equilibrium pressure $P^{H}$. If $P^{V L E} \neq P^{H}$, the quantity $d m_{w}^{i}$ is not right (wrong mass balance), and needs to be fine tuned in order to obtain the "same" pressures. The criterion used is the following:

$$
F_{o b j}\left(d m_{i}\right)=\frac{P^{V L E}-P^{L H E}}{P^{V L E}} \rightarrow 0 \quad\left(<10^{-3}\right)
$$

In order to simplify the calculations, an hypothesis is added: the amount of gas molecules into the liquid phase is considered constant in the calculation of each iterations. This hypothesis is not very strong since the gas solubility of hydrocarbon is generally low, or is slightly impacted by the evolution of gas phase composition at each iterations. Notice also that the liquid water density is considered constant at $1000 \mathrm{~kg} / \mathrm{m}^{3}$ in our simulations (due to low variations within the range of temperature $0-10^{\circ} \mathrm{C}$ ).

By performing the $n$ calculations, the final state (point $D$ ) is reached, and the hydrate flash calculation is over.

The number $n$ affects the length of the calculations, but also the results. This is investigated later in section 4.4.

\subsubsection{Flash calculation framework with hydrate phase reorganization}

Two situations are considered: a reorganization of the last layer to obtain a thermodynamic equilibrium (Gas-Liquid-Last layer of hydrate crystal), or a completelly homogeneous hydrate phase. Earlier, the last layer being crystallized was at thermodynamic equilibrium with the previous iteration ("begining" of the crystallization). Indeed, cavities occupancy is calculated from iteration $i$ to compute iteration $i+1$. So, the layer $i+1$ of the hydrate crystal has an occupancy at equilibrium with the gas phase composition at iteration $i$.

To have a different situation than this, two solutions are conceivable. First, use as many iterations as possible. This will be investigated in section 4.4. Secondly, the last layer can be reorganized in order to compute a hydrate layer completelly at equilibrium with the gas and liquid composition at each step of the process. This implies another loop on the occupancy factor used at the begining of the hydrate phase calculations (i.e. calculate the amount of molecules in the hydrate phase).

In the end, a complete homogeneous hydrate phase is possible to compute. To do so, the method reorganizing the hydrate can be performed with only one iteration (crystal growth in single step). 


\section{Phase reorganization of last layer.}

This second framework (framework II) presents the same hydrate flash calculation than before with the last crystal layers at equilibrium with the liquid phase. The previous framework had to be changed to take into account this reorganization at the end of each step. As the hydrate composition is slightly different with this new model, the final state is also different in terms of mass balance, final pressure and hydrate volume. Though, these two frameworks (I and II) are equilivalent when $n \rightarrow \infty$.

In framework II, a new loop is introduced. In the end of each step the occupancy factor before crystallization is compared with the occupancy factor after. This means that the occupancy factor of the last layer is in agreement with equation 9 according to the liquid state. If there is a difference, the iteration calculation starts again with the previous $\theta_{j}^{i}$ as the starting point (another weighted calculation of the new $\theta_{j}^{i}$ can be utilized to improve the algorithm speed). The calculation of the amount of water crystallized $\left(d m_{w}^{i}\right)$ is repeated with a new gas consumption until $\left(\theta_{i}^{j}\right)_{\text {initial }}=\left(\theta_{i}^{j}\right)_{\text {final }}$. Figure 4 describes framework II.

\section{Homogeneous hydrate phase.}

In this approach (framework II*) the whole hydrate phase is at equilibrium with the liquid phase, both from local and global points of view. The first possibility to obtain this is to use the second framework, and use only one iteration from framework II. This can cause a problem of convergence since only one iteration implies an initial state far from the final equilibrium (hard to reach). Another solution is to use the total mass of the hydrate phase $\left(m_{w}^{H}\right)$ instead of the new amount $d m_{w}^{H}$. This divides the calculations into successives $m_{w i}^{H}$ crystallized, and avoids convergence issues. As a consequence (many loops), the computing time of this approach is slightly higher. More work on the algorithm would optimize the calculations.

Figure 5 shows the path of framework II*. 


\section{Simulation results}

The two frameworks are tested and compared to reference results. These results are from a previous experimental work [11] (from $\mathrm{CO}_{2}, \mathrm{CH}_{4}, \mathrm{C}_{2} \mathrm{H}_{6}$ gas mixture).

The thermodynamic frameworks were computed through a MATLAB code. Since it is a "new code", Kihara parameters optimization was necessary. Indeed, the Kihara parameters are very author (and code)-dependents.

Then, the frameworks were compared to experimental results. A great deal of attention was paid to the number of iterations $n$.

Afterwards, a parametric study of kihara parameters is suggested to determine the consequences of the uncertainty of kihara parameters on the equilibrium pressure and hydrate volume.

Finally, the results are analysed looking at the experimental results, both at quick and slow crystallization, to discuss the hydrate crystallization process.

\subsection{Kihara parameters}

A set of experimental pure hydrates equilibria was considered ( 8 data from $[26,27]$ for $\mathrm{CO}_{2}, 14$ data from $[26,11,28,29,30]$ for $\mathrm{CH}_{4}$, and 18 data from $[30,31,32,33]$ for $\mathrm{C}_{2} \mathrm{H}_{6}$ ).

Table 3 summarizes the parameters optimized from the above experimental results on pure gas hydrates (temperature range from $273 \mathrm{~K}$ to $300 \mathrm{~K}$ ). The objective is simply to have a starting set of parameters to work with. A MATLAB code of LHE calculation was performed presented, as presented in section 2. The parameters regression was executed with the use of the lsqnonlin function of Matlab, by considering local minima. The root mean square errors (RMSE) obtained are quite satisfying here (from 0.02 to 2.43).

\subsection{Reference case}

The first reference case is a ternary gas mixture + water. The mixture is $\mathrm{CO}_{2}-\mathrm{CH}_{4}-\mathrm{C}_{2} \mathrm{H}_{6}$ at molar compositions of $5 \% / 92 \% / 3 \%( \pm 0.1)$ respectivelly. The initial temperature of the gas mixture is $285.75 \mathrm{~K}$ and pressure was 30.5 bars. The total volume $\left(V^{R}\right)$ is $2.36 L$. The amount of water added to the mixture is $801.3 \mathrm{~g}$. The reference case is taken from Duyen et al.[11]. 


\subsection{Test of thermodynamic model on benchmark}

Table 4 and figure 6 present the results of the thermodynamic model only (without the mass blance component of the flash calculation) compared to the reference baselines. The table shows well that the thermodynamic model (LHE)with our parameters is pretty accurate (RMSE of 0.31 on the pressure). It also demontrates that a slow crystallization is closer to a thermodynamic equilibrium. Duyen et al.[11] on this same mixture, obtained thermodynamic modeling results closer to this experiment at slow crystallization than at quick crystallization. Although another code was used, our results are in accordance with the afore-mentionned study.

\subsection{Frameworks results, and influence of the number of iterations}

Table 5 shows the results for the first reference results $\left(\mathrm{CO}_{2}-\mathrm{CH}_{4}-\mathrm{C}_{2} \mathrm{H}_{6}\right)$. By cooling this mixture, all the frameworks give a three phases equilibrium (nucleation point) at $280.14 K$ and 42.63 bars. This means that when we cool the reference case down to $280.14 K$, this causes the system to crystallize.

Then, there is a cooling, step by step as a function of the iterations number $(n)$. The number of iterations corresponds to the number of cooling steps from the last temperature (here $280.14 K$, or $T_{B}$ on figure 2 ) to the final fixed temperature $\left(274.5 K\right.$, or $T_{f}$, or $\left.T_{D}\right)$. This also corresponds to the discretization of the crystal growth modeling.

Figure 7 illustrates the experimental and predicted thermodynamic paths for the crystallization process (PT digrams) assuming a SI structure. The first figure $(7(\mathrm{a}))$ shows, for framework I $(n=7)$, the first point of crystallization. The successives evolution of the solid phase and the PT equilibrium curves corresponds to each temperature step. The second figure $(7(b))$ shows the experimental path compared to to predicted (all frameworks). Like the theoretical example (figure 2), as the gas is consumed, the most stabilizing molecules are removed from the fluid phases. That is why the system reaches successive equilibrium with a narrower hydrate zone (the $P T$ curves are higher in pressure at a given temperature).

A quick investigation of the iteration number influence shows that frameworks I and II converge when $n \rightarrow \infty$, as expected. Since $n$ impacts the calculation time, it is of utmost importance in process simulations. A number of 20 iterations to model a continuous crystallization is a satisfying compromise. Keep in mind that there is an experimental uncertainty on pressure and mass measurements. When using 20 iterations for frameworks I and II, 
there is a difference of $0.01 b a r$ in pressure and $0.1 \mathrm{~g}$ in hydrate mass compared to $n \rightarrow \infty$. Logically, framework $\mathrm{II}^{*}$ is not affected by $n$.

Concerning final pressure and water mass in the hydrate phase, both frameworks provide solid results (errors under $5 \%$ for pressure and mass). If a few iterations are used $(n=3)$, the accuracy deteriorates. This can also cause the calculation of negative compositions (too many gas molecules into the hydrate phase, and a negative number in the gas phase). Above 10 iterations, frameworks I and II are pretty similar. Framework II* (total hydrate phase reorganization) predicts very well the final pressure, but not as well the hydrate volume (or the water mass in hydrate). So, reorganizaing the crystal, by comparison to the reference case, improves the accuracy of the pressure but decreases the accuracy of water conversion. In framework II, the gas concumption is higher, leading to more gas molecules in the crystal, a higher hydrate volume, and a lower pressure. Indeed, since the final pressure is lower than initially, the final occupancy of the cavity of the whole homogeneous crystal is lower than the occupancy factor of the first layer of frameworks I and II. In detail, the hydration number is 7.02 (II*) against 7.06 (I and II).

The gas and hydrate phase compositions are also well predicted, although the deviation is higher than the experimental uncertainty.

\subsection{Kihara parameters influence on hydrate volume}

As the volume of hydrate formed is crucial for many applications (pipe plugs, $\mathrm{CO}_{2}$ capture...), knowing the Kihara parameters influence of the model is essential. Since these parameters are very author dependent, an evaluation of their uncertainties on the simulation results is presented here.

The Kihara parameters are the characteristics of the guest molecules, but various values exist. Usually, they need to be determined for each code, hence the authors dependency. But, it is not always an easy task since the experimental data can be missing or inaccurate (see discussion section 2.2.3).

In this work, the influence of the Kihara parameters uncertainties on the final pressure and hydrate volume is suggested. To do this investigation, a statistical approach was employed using Monte Carlo simulations. 1000 random $(\epsilon / k$ and $\sigma)$ parameters were computed according to a normal distribution around the mean values given in table 3). The standard deviations in the normal distribution were taken from $1 \%$ to $5 \%$. These Monte Carlo simulations were performed using the framework I with 8 iterations (to lower time consumption and good approximation of the final state). 
Table 6 presents the effect of kihara parameters uncertainty on the final equilibrium pressure and hydrate volume. This demontrates a small difference in the Kihara parameter value $(5 \%)$ can have a significant consequence on the final pressure and hydrate volume of standard deviations of $42 \%$ and $125 \%$ respectivelly.

Since the models deviations from experimental results are usually under $5 \%$, it can be concluded that the frameworks are in good accordance with the experiments.

\subsection{Understanding hydrate crystallization}

Initially, an experimental reference case at low crystallization rate was chosen. The objective was to compare a thermodynamic based model to an experiment which was as close as possible to a transformation at thermodynamic equilibrium (no kinetic effects).

Towards the better understanding of the clathrate hydrate crystallization, firstly framework I is compared to more usual experimental measurements at quick crystallization rates. These data come from the previous study [11] on $\mathrm{CO}_{2}-\mathrm{CH}_{4}$ and $\mathrm{CO}_{2}-\mathrm{CH}_{4}-\mathrm{C}_{2} \mathrm{H}_{6}$ gas mixtures.

Table 7 gives the results on the final pressures and masses of water in hydrate phase. Surprisingly, the results are in pretty good accordance with the final equilibrium pressure (deviation $<5 \%$ ). Since we suspect a kinetic effect on the hydrate crystallization, a higher difference was expected. Concerning the final volume of hydrate (or final mass of water in hydrate phase), the deviation from the experimental results is higher, but within the 3\% uncertainty ( \pm standard devivation $\sigma$ ) on the kihara parameters (or within $1 \%$ uncertainty at \pm variance $/ 2 \sigma)$.

In conclusion, it seems that the $\mathrm{CO}_{2} / \mathrm{CH}_{4}$ gas mixtures are not very much affected by kinetics. Also, framework I with 5 iterations gives slighlty better results. This framework, combined with this number of iterations, was not the best as compared to the reference case at slow driving force. This could validate that a higher driving force is closer to framework I with no hydrate reorganization. Nevertheless, the results are more than adequate, and it would probaly not be the same for other mixtures where kinetics could be predominating. 


\section{Conclusion}

Two main thermodynamic frameworks for the clathrate hydrate crystallization have been suggested. They are thermodynamic flash calculations of three phases equilibria (aquaeous, gas and hydrate) at constant volume. The temperature is the variable imposed. Two thermodynamic paths have been modelled and investigated.

In frameworks I and II, the crystallization is discretized in mass of hydrate (equivalent to subcooling degree). Crystal growth is supposed to occur at local thermodynamic equilibrium with the surrounding liquid (itself at thermodynamic equilibrium with the surrounding gas phase). In the case of framework I, the equilibrium is considered before crystallization, and after for framework II. The discretization corresponds to successives layers of the hydrate crystal. As a consequence, the final clathrate is non-stoichiometric (local composition different than whole composition).

In a slightly different physical approach with a single iteration (framework $\left.\mathrm{II}^{*}\right)$, the local composition of the hydrate phase is the same as the overall composition (homogeneous solid phase in composition). To accomplish this, the crystal composition is homogenized during crystallization.

The simulation results, compared to a reference case (experiment at low crystallization rate from $\mathrm{CO}_{2}-\mathrm{CH}_{4}-\mathrm{C}_{2} \mathrm{H}_{6}$ gas mixture), are fairly accurate. All the frameworks show a deviation on the final pressure and mass of crystallized water under 5\%. Frameworks I and II provide better results in terms of crystallized mass of water while framework $\mathrm{II}^{*}$ seems to be better at predicting the final pressure. Also, a study on the uncertainties due to Kihara parameters uncertainties showed that the experimental results are within the margin of error for the models.

Concerning the discretization of the crystallization, a number above 20 iterations for the crystal growth is enough (frameworks I and II). As suspected, frameworks I and II converge when the iteration number is infinite.

The influence of the uncertainties on Kihara parameters has been studied on framework I. It has shown that the results are very sensitive to these kind of errors (errors of $1 \% \Rightarrow 15 \%$ on Pressure and $35 \%$ on hydrate volume).

It is important to notice and underline that these flash calculations are only based on thermodynamic equilibria. If different paths are investigated, then no kinetic considerations exist such as mass transfer limitation at gas/liquid interface[34], or possible diffusion effects at the liquid/hydrate interface[12]. These considerations might be necessary to model hydrate crystallization at 
613

high driving force, where the final pressure could to be significantly affected. These frameworks provide a usefull tool for industry and academia to predict with a more reallistic accuracy the final pressure, as well as give a good order of magnitude of the hydrate volume. For gas mixtures that do not show important kinetic effects (such as $\mathrm{CO}_{2} / \mathrm{CH}_{4}$, as investigated in this study), predictions are still accurate and within the same uncertainties.

Future works might include further elaboration of the principles discovered while at the same time simplifying the process so that it can be clearly implicated in industrial situations.

\section{Acknowledgment}

The authors would like to thank Christopher Yukna very much for English advice and correction 
${ }_{625}$ List of symbols 


\begin{tabular}{|c|c|c|}
\hline Symbol & description & dimension \\
\hline$a$ & hard core radius & $n m$ \\
\hline$a_{w}^{L}$ & liquid water activity & - \\
\hline$C$ & Langmuir constant & $n m^{3} / J$ \\
\hline$c_{p}$ & Molar calorific capacity & $\mathrm{J} / \mathrm{mol} / \mathrm{K}$ \\
\hline$f$ & Fugacity & $P a$ \\
\hline$K_{H, i, w}$ & Henry's constant & $P a$ \\
\hline$k_{B}$ & Boltzmann constant & $J / K$ \\
\hline$h$ & molar enthalpy & $J / m o l$ \\
\hline$m$ & total mass of water & $g$ \\
\hline$m_{i}$ & initial mass of water & $g$ \\
\hline$n$ & number of iterations in frameworks (growth discretization) & - \\
\hline$N$ & number of experimental data & - \\
\hline$P$ & Pressure & bars \\
\hline$T$ & Temperature & ${ }^{\circ} \mathrm{C}$ or $\mathrm{K}$ \\
\hline$V_{m, i}^{\infty, L_{w}}$ & Partial molar volume of $i$ at infinite dillution & $\mathrm{cm}^{3} / \mathrm{mol}$ \\
\hline$w$ & interaction potential & $J$ \\
\hline$x$ & Molar composition of compound in hydrate phase & mol.frac. \\
\hline$Z$ & Compressibility factor & \\
\hline$\epsilon$ & maximum attractive potential & $J$ \\
\hline$\nu_{w}$ & Water molar volume & $m^{3} / \mathrm{mol}$ \\
\hline$\nu_{i}$ & number of cavity $i$ per mole of water & $\mathrm{mol} / \mathrm{mol}$ \\
\hline$\mu$ & Chemical potential & $J / m o l$ \\
\hline$\rho$ & Density & $\mathrm{kg} / \mathrm{m}^{3}$ \\
\hline$\sigma$ & distance from cavity center & $n m$ \\
\hline$\theta_{j}^{i}$ & Occupancy factor of molecule $j$ in cavity $i$ & - \\
\hline$\phi$ & Fugacity coefficient & \\
\hline Superscript & description & \\
\hline 0 & reference state & \\
\hline$\beta$ & Empty hydrate reference state & \\
\hline$H$ & Hydrate phase & \\
\hline$j$ & molecule index & \\
\hline$L$ & Liquid phase & \\
\hline$L_{w}$ & Aqueous liquid phase & \\
\hline$V$ & Vapor phase & \\
\hline Subscript & description & \\
\hline $\exp$ & experimental result & \\
\hline$i$ & iteration number, or intial state, or index of cavity $(1,2,3)$ & \\
\hline$m$ & molar quantity & \\
\hline pred & predicted result & \\
\hline & water molecule & \\
\hline
\end{tabular}


Table 1: Thermodynamic properties of liquid water compared to $\beta$ structure

\begin{tabular}{|c|c|c|c|c|}
\hline Structure & & Structure & & \\
\hline$\Delta \mu_{w}^{L-\beta, 0}$ & $\Delta h_{w}^{I-\beta, 0}$ & $\Delta \mu_{w}^{L-\beta, 0}$ & $\Delta h_{w}^{I-\beta, 0}$ & \\
\hline 699 & 0 & 820 & 0 & van der Waals and Platteuw [21] \\
\hline 1255.2 & 753 & 795 & 837 & Child [35] \\
\hline 1297 & 1389 & 937 & 1025 & Dharmawardhana et al. [36] \\
\hline 1120 & 931 & 1714 & 1400 & John et al. [37] \\
\hline 1287 & 934 & 1068 & 764 & Handa and Tse [23] \\
\hline
\end{tabular}

\section{${ }_{626}$ Tables}


Table 2: Reference properties of hydrates from Sloan[2]

\begin{tabular}{|c|c|c|c|}
\hline & unit & Structure I & Structure II \\
\hline$\Delta h_{w}^{L-\beta, 0}$ & $\mathrm{~J} / \mathrm{mol}$ & $\Delta h_{w}^{I-\beta} \mid T^{0}, P^{0}, S I-6011$ & $\Delta h_{w}^{I-\beta} \mid T^{0}, P^{0}, S I I-6011$ \\
\hline$\left.\Delta \nu_{w}^{L-\beta}\right|_{T^{0}, P^{0}}$ & $10^{-6} \mathrm{~m}^{3} / \mathrm{mol}$ & 4.5959 & 4.99644 \\
\hline$\left.\Delta C_{p_{w, m}}^{L-\beta}\right|_{T^{0}, P^{0}}$ & $J / \mathrm{mol} / \mathrm{K}$ & -38.12 & -38.12 \\
\hline$b_{p, w}^{L-\beta}$ & $J / \mathrm{mol} / \mathrm{K}^{2}$ & 0.141 & 0.141 \\
\hline
\end{tabular}


Table 3: Quick Kihara parameters optimization of $\mathrm{CO}_{2}, \mathrm{CH}_{4}$, and $\mathrm{C}_{2} \mathrm{H}_{6}$ on a few experimental data[27, 26, 11, 28, 29, 30, 31, 32, 33] (* data from Sloan et al. [2])

\begin{tabular}{llllll} 
& $\epsilon / k$ & $\sigma$ & $a^{*}$ & RMSE & nb. of data \\
\hline $\mathrm{CO}_{2}$ & 171.78 & 2.978 & 0.6805 & 0.02 & 8 \\
$\mathrm{CH}_{4}$ & 160.70 & 3.101 & 0.3834 & 2.43 & 14 \\
$\mathrm{C}_{2} \mathrm{H}_{6}$ & 176.83 & 3.22 & 0.5651 & 0.062 & 18 \\
\hline
\end{tabular}




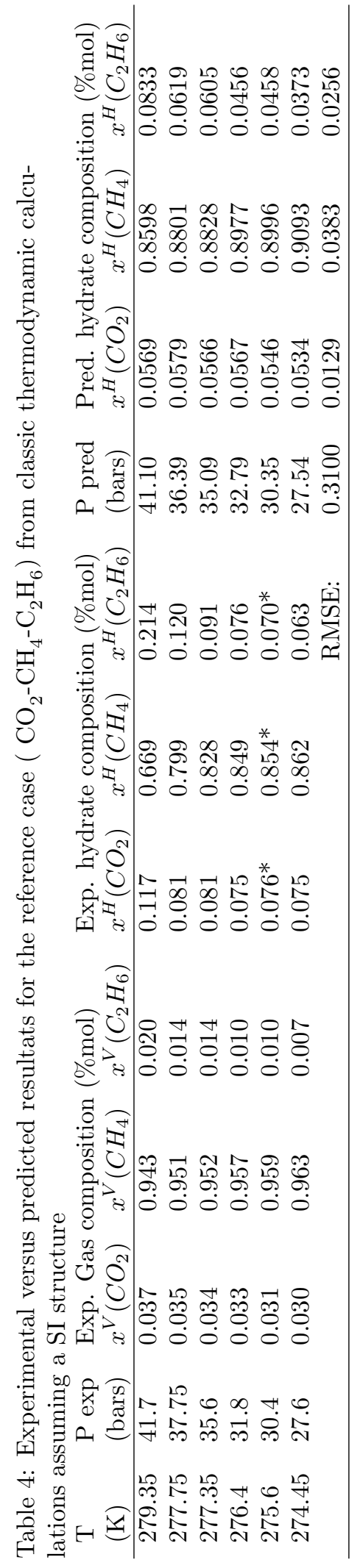




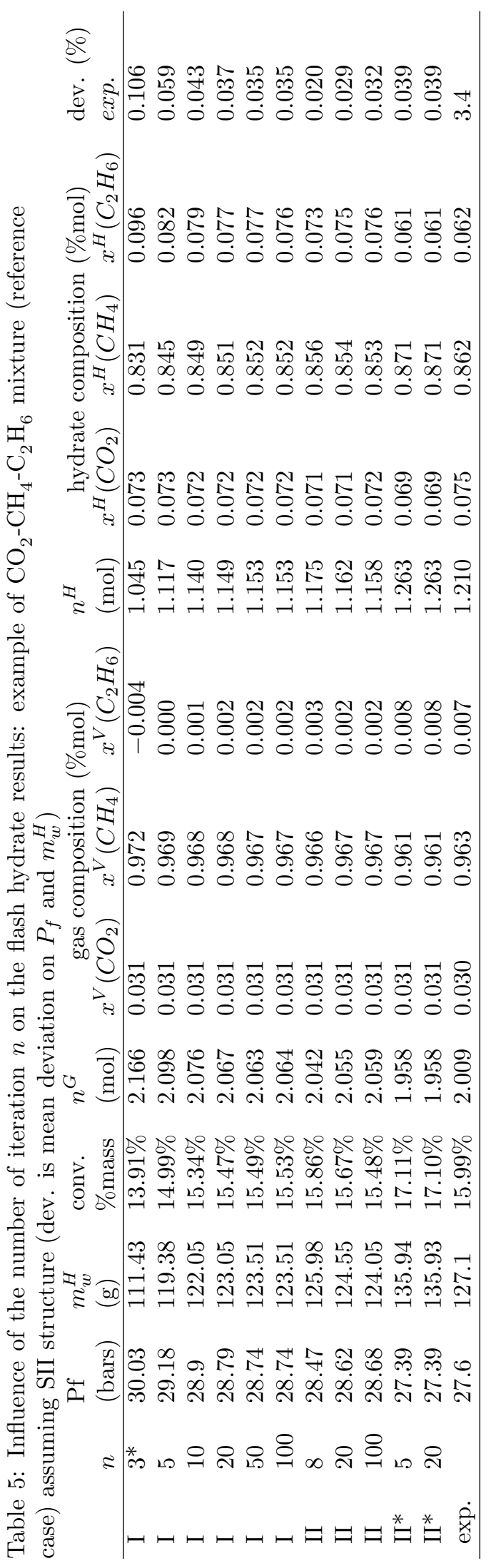


Table 6: Influence of the Kihara parameters uncertainties on the flash hydrate results (framework I, reference case)

\begin{tabular}{lllclll} 
& $\bar{P}_{f}$ & \multicolumn{2}{c}{$\operatorname{std}\left(P_{f}\right)$} & \multicolumn{2}{c}{$\overline{V^{H}}$} & \multicolumn{2}{c}{$\operatorname{std}\left(V^{H}\right)$} \\
uncert. & (bars) & (bars) & $\%$ & $(\mathrm{~mL})$ & $(\mathrm{mL})$ & \multicolumn{1}{c}{$\%$} \\
\hline $1 \%$ & 30.6 & 4.7 & $15.36 \%$ & 162 & 56 & $34.57 \%$ \\
$2 \%$ & 31.204 & 8.06 & $25.83 \%$ & 152.3 & 98 & $64.35 \%$ \\
$3 \%$ & 31.55 & 10.4 & $32.96 \%$ & 145.7 & 128.5 & $88.19 \%$ \\
$5 \%$ & 32.05 & 13.4 & $41.81 \%$ & 138.6 & 172.9 & $124.75 \%$ \\
\hline
\end{tabular}


Table 7: Framework I predictions $(n=20)$ compared to experimental results[11] at quick crystallization (gas 3-4 from $\mathrm{CO}_{2}-\mathrm{CH}_{4}$ mixtures and 6-7-8 from $\mathrm{CO}_{2}-\mathrm{CH}_{4}-\mathrm{C}_{2} \mathrm{H}_{6}$ mixtures) and slow crystallization (reference case of the present study)

Framework I, $n=20$

\begin{tabular}{|c|c|c|c|c|c|c|c|c|}
\hline $\begin{array}{l}\text { Gas } \\
\text { mixtures }\end{array}$ & $\begin{array}{l}T_{f} \\
\left( \pm 0.1^{\circ} \mathrm{C}\right)\end{array}$ & $\begin{array}{l}P_{f} \\
( \pm 0.1 \text { bars })\end{array}$ & $\begin{array}{l}V^{H} \\
(m L \pm 4 \%)\end{array}$ & $\begin{array}{l}m^{H} \\
(g \pm 4 \%)\end{array}$ & $\begin{array}{l}P_{f_{\text {pred }}} \\
\text { (bars) }\end{array}$ & $m_{\text {pred }}^{H}$ & $\begin{array}{l}\operatorname{dev} P \\
(\%)\end{array}$ & dev $m^{H}$ \\
\hline Gas 3 & 3.4 & 33.3 & 268.4 & 212.0 & 31.6 & 256 & 5 & 21 \\
\hline Gas 4 & 2.2 & 29.1 & 217.8 & 172.1 & 28.19 & 186.11 & 3.1 & 8.2 \\
\hline Gas 6 & 2.2 & 30.7 & - & - & 31.59 & 139.9 & 2.9 & - \\
\hline Gas 7 & 1 & 27.1 & - & - & 26.21 & 43.1 & 3.3 & - \\
\hline Gas 8 & 2.75 & 35.4 & 345.3 & 272.8 & 33.87 & 333.5 & 4.3 & 22 \\
\hline Gas 12 & 1.3 & 27.6 & 160.9 & 127.1 & 28.79 & 123.1 & 4.3 & 3.2 \\
\hline & \multicolumn{4}{|c|}{ Framework I, $n=5$} & \multicolumn{4}{|c|}{ Framework II* } \\
\hline $\begin{array}{l}\text { Gas } \\
\text { mixtures }\end{array}$ & $\begin{array}{l}P_{f} \text { pred } \\
\text { (bars) }\end{array}$ & $\begin{array}{l}m^{H}{ }_{\text {pred }} \\
(\mathrm{g})\end{array}$ & $\begin{array}{l}\operatorname{dev} P \\
(\%)\end{array}$ & $\begin{array}{l}\operatorname{dev} m^{H} \\
(\%)\end{array}$ & $\begin{array}{l}P_{f_{\text {pred }}} \\
(\text { bars })\end{array}$ & $\begin{array}{l}m^{H}{ }_{\text {pred }} \\
(\mathrm{g})\end{array}$ & $\begin{array}{l}\operatorname{dev} P \\
(\%)\end{array}$ & $\begin{array}{l}\operatorname{dev} m^{H} \\
(\%)\end{array}$ \\
\hline Gas 3 & 31.7 & 255.5 & 5 & 21 & 31.3 & 259 & 5.9 & 22 \\
\hline Gas 4 & 28.26 & 185.4 & 2.9 & 7.8 & 28.1 & 187.9 & 3.4 & 9.2 \\
\hline Gas 6 & 32 & 135.9 & 4.2 & - & 29.95 & 155 & 2.4 & - \\
\hline Gas 7 & 26.2 & 43 & 3.3 & - & 25.53 & 49.3 & 5.8 & - \\
\hline Gas 8 & 33.88 & 333.1 & 4.3 & 22 & 32.3 & 349.5 & 8.8 & 28 \\
\hline Gas 12 & 29.2 & 119.4 & 5.8 & 6.1 & 27.39 & 135.9 & 0.08 & 6.9 \\
\hline
\end{tabular}




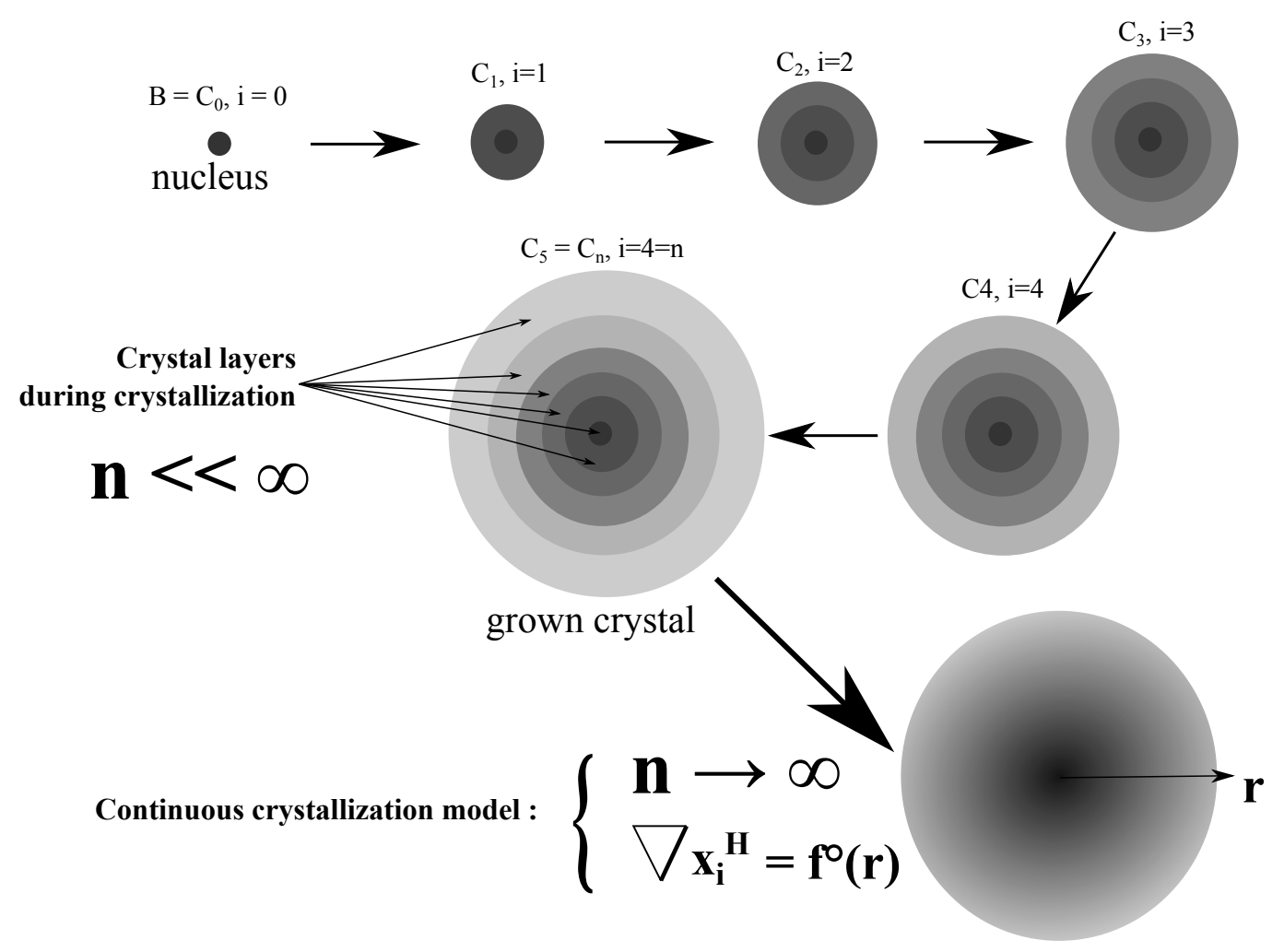

Figure 1: Non-stoichiometric clathrate hydrate growth model (layer by layer growth at different successive equilibrium) 


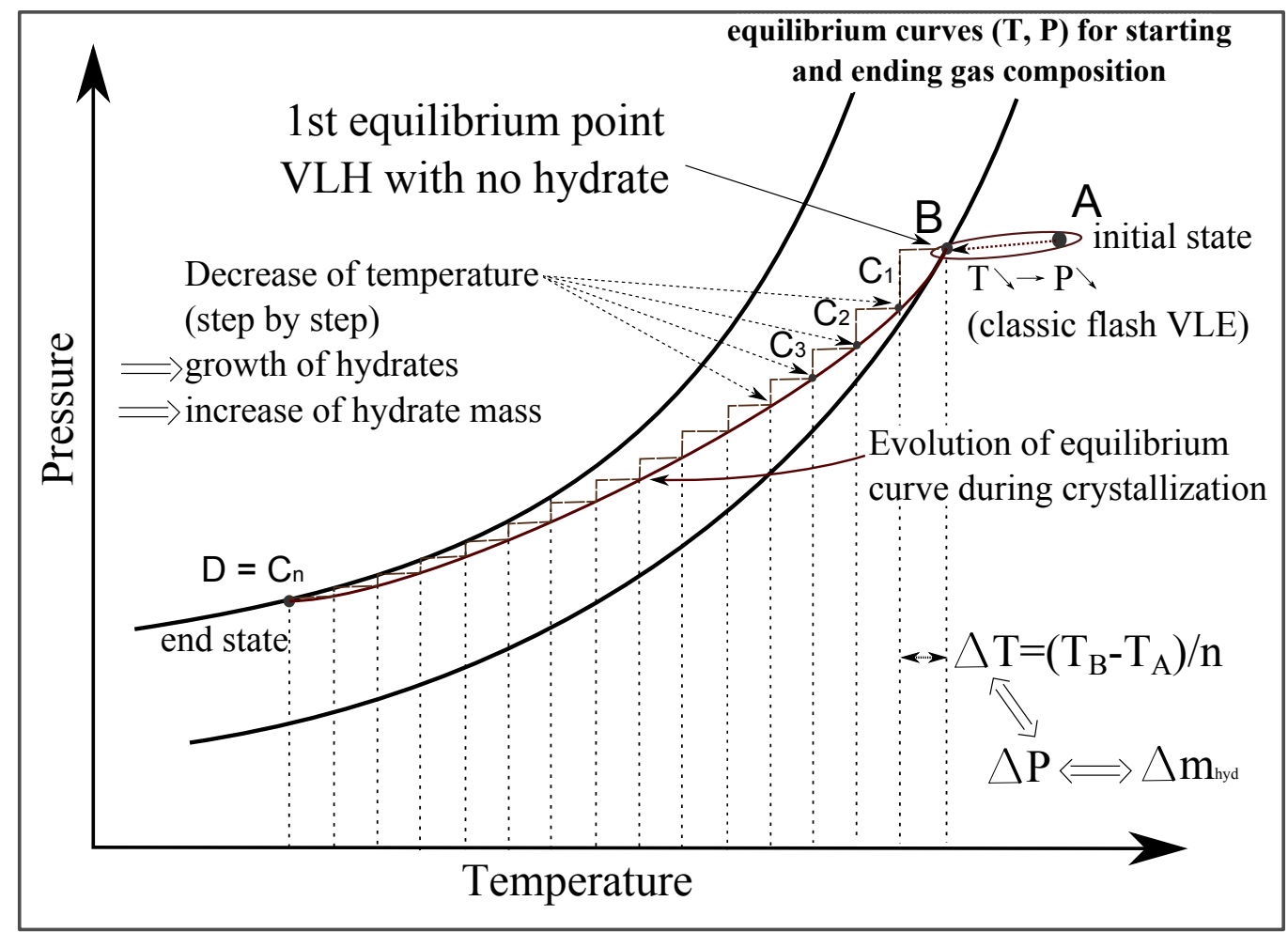

Figure 2: PT evolution during crystal growth of non-stoichiometric hydrate from the intial state (A point), to the 1st three phases equilibrium (B point) and the successives equilibrium during crystallization $\left(C_{i}\right.$ and $\mathrm{D}$ points). 


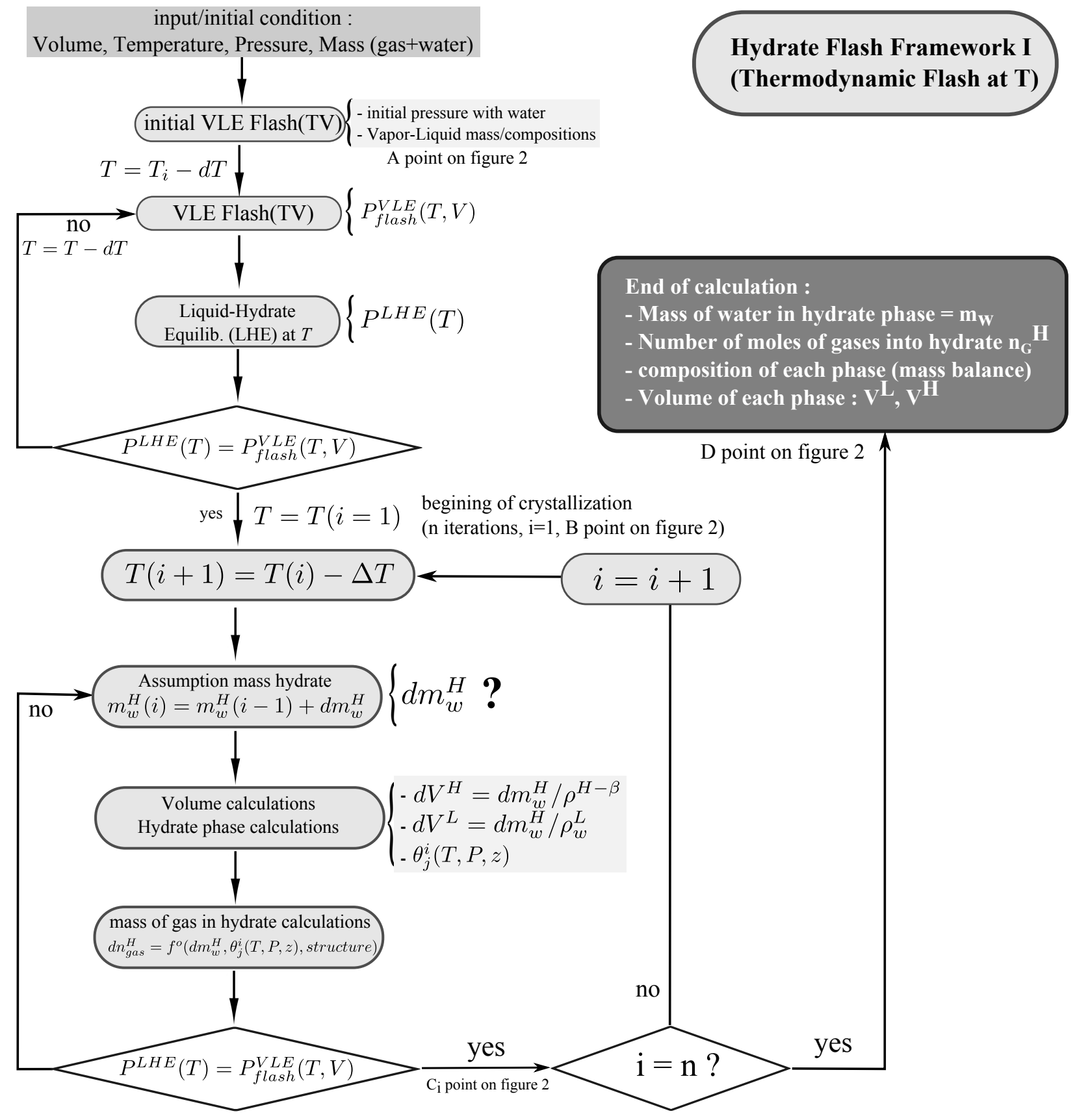

Figure 3: Non-stoichiometric flash hydrate framework: discretization of the cooling in $\Delta T$ steps, assumption of the mass of hydrate, validation of the VLH equilibrium 


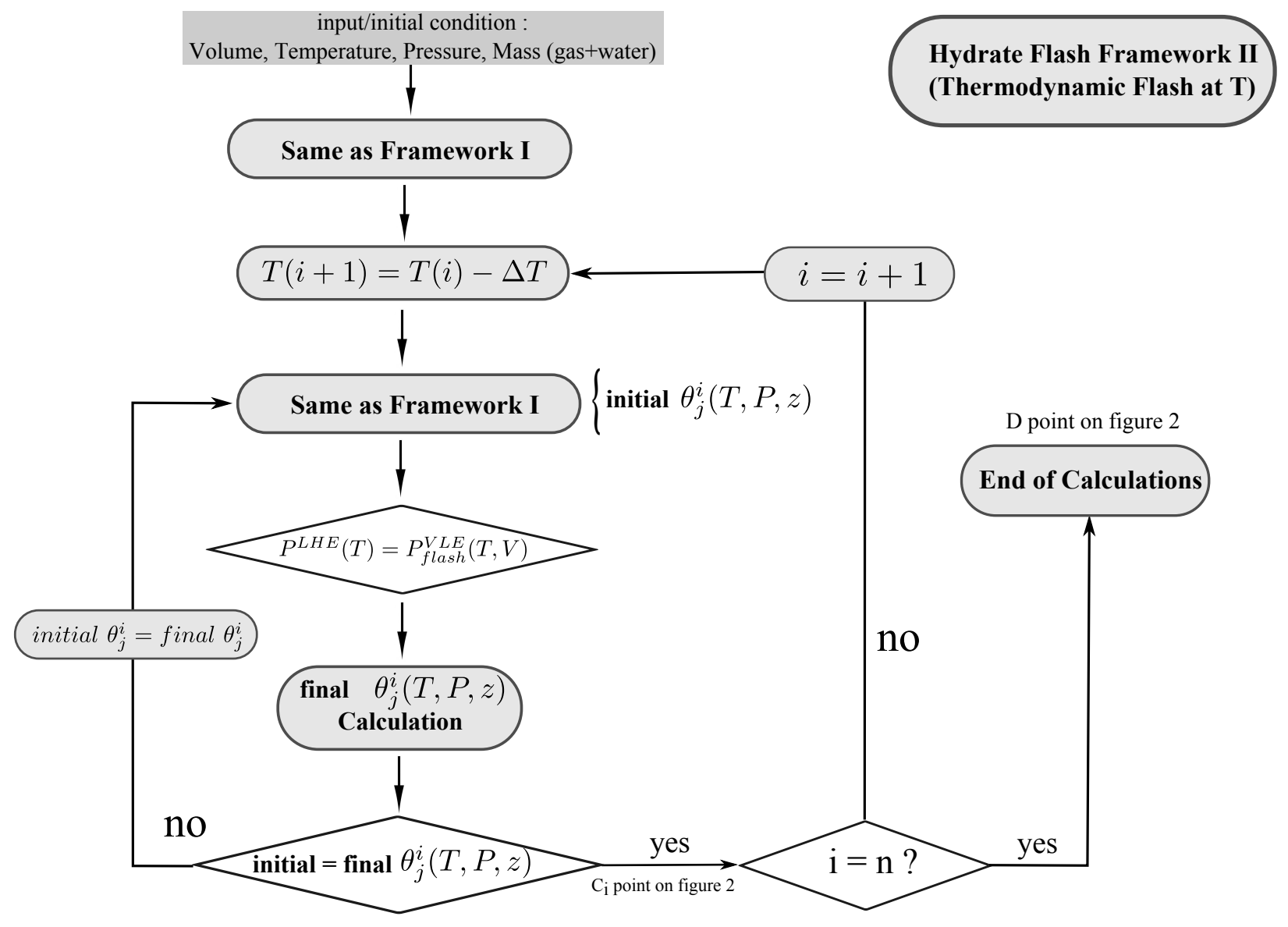

Figure 4: Flash hydrate framework II with reorganization of hydrate last layer, or "stoichiometric" flash hydrate framework II* (homogeneous phase at final gas composition = unique occupancy factor) if $n=1$ 




Figure 5: Clathrate hydrate growth approach with hydrate phase reorganization (framework $\mathrm{II}^{*}$ ) for 4 iterations 


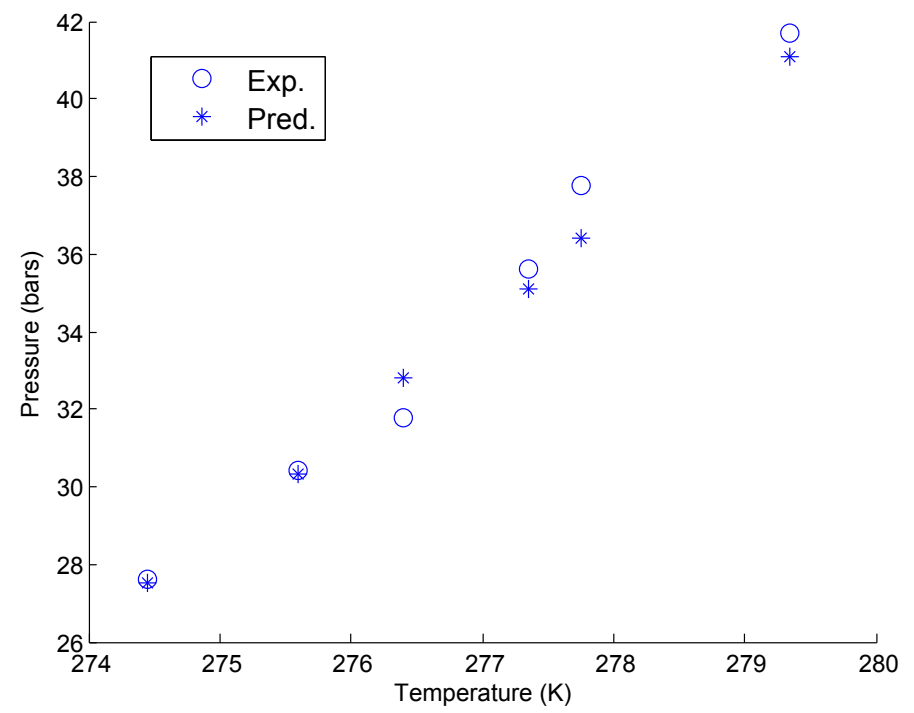

Figure 6: PT diagram of experimental and predicted results of the reference case $\left(\mathrm{CO}_{2}-\right.$ $\mathrm{CH}_{4}-\mathrm{C}_{2} \mathrm{H}_{6}$ ) at different temperatures from the same initial state (see section 4.2) 


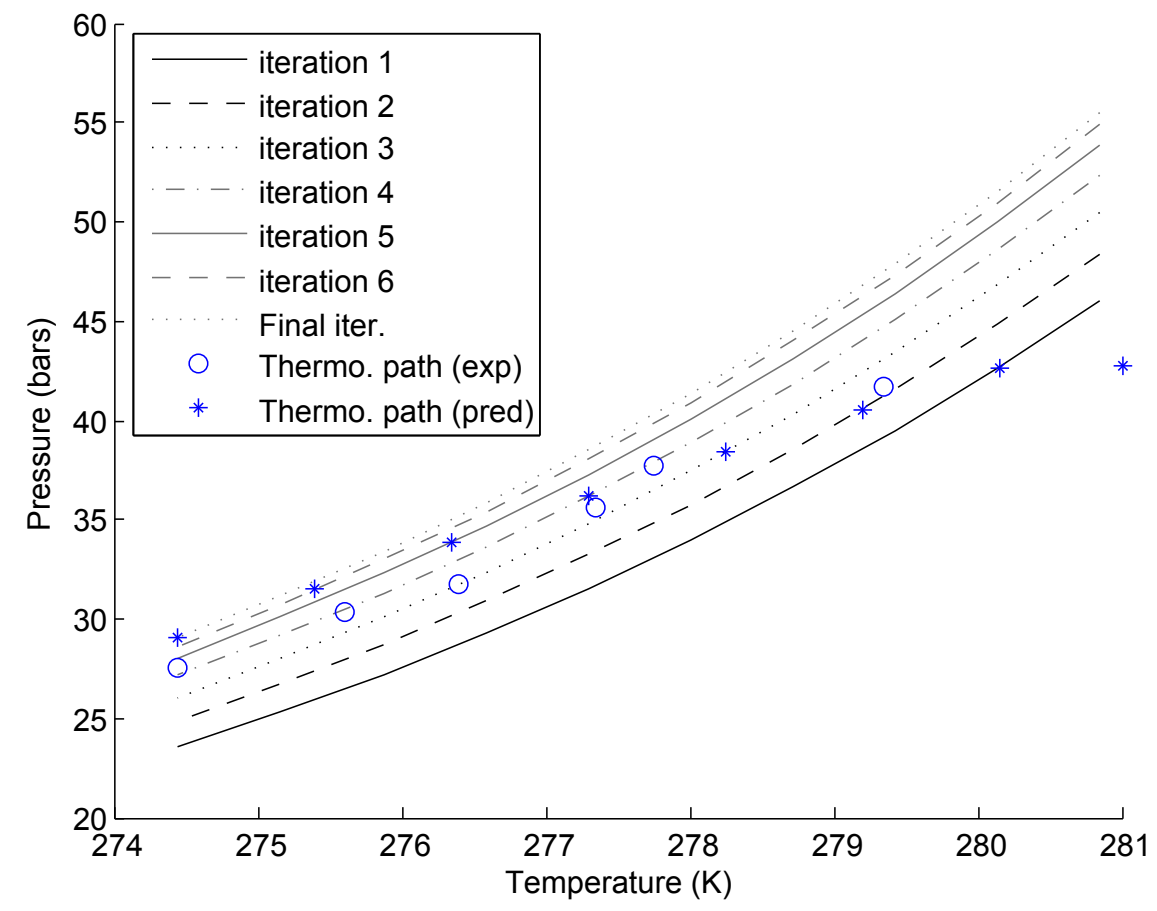

(a) Experimental and framework I $(n=7)$

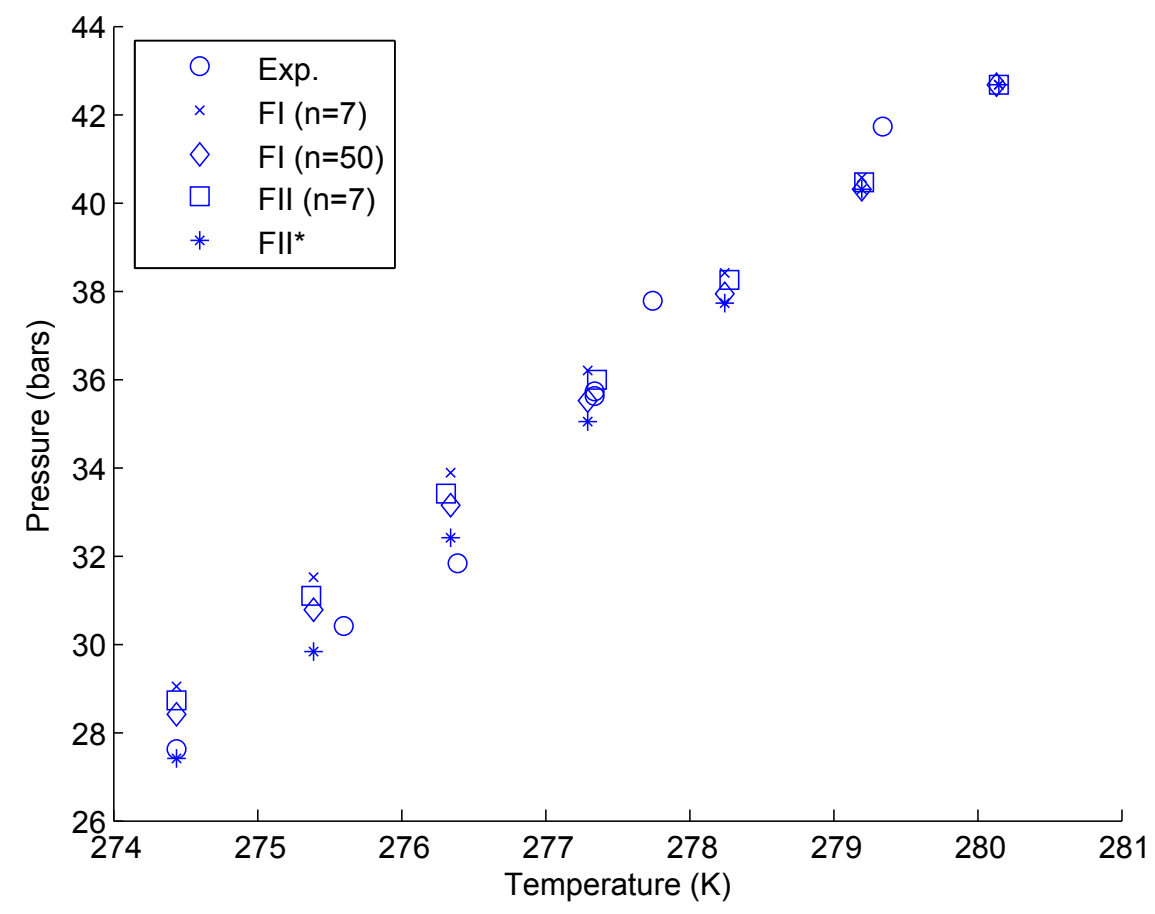

(b) Experimental and all frameworks during crystallization (PT)

Figure 7: Predicted and experimental thermodynamic paths (Pressure vs temperature during crystallization) in the reference case (all frameworks at different numbers of iterations) 


\section{References}

[1] H. T. Falenty, A., W. Kuhs, Formation and properties of ice xvi obtained by emptying a type sii clathrate hydrate, NATURE 516 (2014) 231.

[2] E. Sloan, C. Koh, Clathrate Hydrates of Natural Gases, 3rd ed., CRC Press, Boca Raton, 2007.

[3] G. Jeffrey, "Hydrate inclusion compounds": in Atwood, J. L.; Davies, J. E. D.; MacNicol, D. D.; eds., "Inclusion compounds", I,, Vol. I, Academic Press, New York, 1984.

[4] F. E. Deaton, W.M., Gas hydrates and their relation to operation of natural-gas pipelines, U.S., Bur. Mines Monogr. 8 (1946) 1-101.

[5] L. K. Mokhatab S., Wilkens RJ., A review of strategies for solving gashydrate problems in subsea pipelines, Energy Sources. Part A: Recovery. Utilization. and Environmental Effects 29.

[6] C. F. Duc, N.H., J. Herri, Co2 capture by hydrate crystallization a potential solution for gas emission of steelmaking industry, Energy Conversion Management 8 (2007) 1313-1322.

[7] K. M. L. A. C. F. F. D. Douzet, J., J. Herri, Prototyping of a real size air-conditioning system using a tetra-n-butylammonium bromide semiclathrate hydrate slurry as secondary two-phase refrigerant - experimental investigations and modelling, International Journal of RefrigerationRevue Internationale du Froid 36.

[8] J. Herri, C. E., Carbon dioxide, argon, nitrogen and methane clathrate hydrates: thermodynamic modeling, investigation of their stability in martian atmospheric conditions and variability of methane trapping, Planetary and Space Science 73 (2012) 376-386.

[9] K. kvenvolden, A review of the geochemistry of methane in natural gas hydrate, Organic Geochemistry 23.

[10] E. Sloan, A changing hydrate paradigm-from apprehension to avoidance to risk management, Fluid Phase Equilibria 228-229 (2005) 67-74. 
[11] L. Q. D. B. B. H. J. G. P. Le Quang, D., P. Duchet-Suchaux, Experimental procedure and results to measure the composition of clathrate hydrates, during crystallization and at quilibrium, from $n_{2}-\mathrm{CO}_{2}-\mathrm{ch}_{4}-c_{2} h_{6^{-}}$ $c_{3} h_{8}-c_{4} h_{10}$ gas mixtures, Submitted to Fluid Phase Equilibria.

[12] J. Herri, M. Kwaterski, Derivation of a langmuir type model to describe the intrinsic growth rate of gas hydrates during crystallization from gas mixtures, Chemical Engineering Science 81 (2012) 28-37.

[13] S. r. H. Boesen, R.R., K. Perdersen, New approach for hydrate flash calculations, Proceedings of the 8th International Conference on Gas Hydrates.

[14] P. Skovborg, P. Rasmussen, A mass transport limited model for the growth of methane and ethane gas hydrates, Chemical Engineering Science 49 (1994) 1131-1143.

[15] G. Soave, Equilibrium constants from a modified redlich-kwong equation of state, Chemical Engineering Science 27 (1972) 1197-1203.

[16] A. Danesh, PVT and Phase Behavior of Petroleum Reservoir Fluids, Elsevier, 1998.

[17] J. Prausnitz, R. Lichtenthaler, E. de Azevedo, Molecular thermodynamics of fluid-phase equilibria, Prentice Hall PTR, 1998.

[18] B. A. K. M. F. A. O. Y. Herri, J.M., C. A., Gas hydrate equilibria from co2-n2 and co2-ch4 gas mixtures, - experimental studies and thermodynamic modeling, Fluid Phase Equilibria 301 (2011) 171-190.

[19] G. Holder, S. Zetts, N. Pradhan, Phase behavior in systems containing clathrate hydrates: A review, Reviews in Chemical Engineering 5.

[20] E. Sloan, Clathrate Hydrates of Natural Gases, 2nd ed., Marcel Dekker, New York., 1998.

[21] P. J. van der Waals, J.H., Clathrate solutions, Adv. Chem. Phys. 2 (1959) 1-57.

[22] M. H. von Stackelberg, M., On the structure of gas hydrates, J. Chem. Phys. 2 (1951) 1319-1320. 
[23] T. J. Handa, Y.P., J. Phys. Chem. 23 (1986) 5917.

[24] V. McKoy, O. J. SinanoĞlu, Theory of dissociation pressures of some gas hydrates, J Chem. Phys 38 (1963) 2946-2956.

[25] L. Michelsen, J. Mollerup, Thermodynamic models: Fundamentals \& Computational Aspects, 2nd edition, Tie-Line Publications, 2007.

[26] F. R. Adisasmito, S., E. Sloan, Hydrate of carbon dioxide and methane mixtures, J. Chem. Eng. Data 36 (1991) 68-71.

[27] S. Larson, Phase studies of the two component carbon dioxide-water system involving the carbon dioxide hydrate, Ph.D. thesis, University of Illinois, Urbana, IL (1955).

[28] Y. Dyadin, E. Aladko, Proceedings of the 2nd Int.Conf.on Natural Gas Hydrates, Toulouse.

[29] H. G. Thakore, J.L., Solid vapor azeotropes in hydrate-forming systems, Ind. Eng. Chem. Res. 26 (1987) 462-469.

[30] O. R. Yasuda, K., Phase equlibrium of clathrate hydrates formed with methane, ethane, propane or carbon dioxyde at temperatures below the freezing point of water, J. Chem. Eng. Data 53 (2008) 2182-2188.

[31] D. A. Avlonitis, D., A. Todd, Measurement and prediction of hydrate dissociation pressure of oil-gas systems, Ph.D. thesis, Heriot-Watt University, Edinburgh (1988).

[32] B. P. R. Englezos, P., Experimental study on the equilibrium ethane hydrate formation conditions in aqueous electrolyte solutions, Ind. Eng. Chem. (30) 1655-1659.

[33] O. L. Nixdorf, J., Experimental determination of hydrate equilibrium conditions for pure gases, binary and ternary mixtures and natural gases, Fluid Phase Equilib. 139.

[34] P. J.-S. G. F. Herri, J.M., M. Cournil, Interest of in situ particle size distribution for the characterization of methane hydrate formation: Identication of the mechanisms of crystallization, AIChE Journal 45 (1999) 590-602. 
717

[35] W. J. Child, Thermodyamic functions for metastable ice structures i and ii, J. Phys. Chem. 68 (1964) 1834-1838.

[36] P. Dharmawandhana, The measurement of the thermodynamic parameters of the hydrate structure and application of them in the prediction of natural gas hydrates, Ph.D. thesis, Colorado School of Mines (1980).

[37] P. K. John, V.T., G. Holder, AIChE. J. 31 (1985) 252-259. 